\title{
El emprendimiento y la potencialidad de los alumnos de la Universidad Nacional Mayor de San Marcos
}

ANdrea Teresa de Jesús CONDORI GONZÁLEZ Pedro Pablo Rosales López ${ }^{2}$

RECIBIDO: 20/07/2018 ACEPTADO: 03/10/2018

\begin{abstract}
RESUMEN
El siguiente trabajo tiene como finalidad dar a conocer el panorama del emprendedurismo en América Latina, desde sus variables internas; como el perfil promedio del emprendedor, hasta las externas, que afecten directamente el modelo. También se busca incrementar el conocimiento que el emprendedor debe considerar para que su proyecto se haga realidad e incremente sus fortalezas y competitividad. Finalmente, se ha elaborado un estudio sobre una muestra de alumnos de la Universidad Nacional Mayor de San Marcos y su análisis respectivo para revelar su potencialidad con respecto al emprendimiento.
\end{abstract}

Palabras-claves: Emprendimiento; Impacto del emprendimiento; Perfil del emprendedor.

ENTREPRENEURSHIP AND POTENTIAL OF UNIVERSIDAD

Nacional Mayor de San Marcos StUdents

\section{ABSTRACT}

The purpose of the following paper is to provide information on entrepreneurship situation in Latin America, starting from its internal variables, such as the average entrepreneur profile, to external variables that could directly affect the model. It also aims to increase the knowledge entrepreneurs should consider in order to realize their projects and increase their strengths and competitiveness. Finally, a study on a sample of students from the Universidad Nacional Mayor de San Marcos was conducted and analyzed in order to reveal student's potential regarding entrepreneurship.

Keywords: Entrepreneurship; entrepreneurial impact; entrepreneur profile.

\section{INTRODUCCIÓN}

En los últimos años se han visualizado diferentes respuestas con cambios radicales en el panorama económico global. Desde la crisis internacional hasta los conflictos sociales han sido determinantes para encausar de diversas modalidades las estrategias de negocio de los grupos económicos a nivel macro y micro. Es a razón de la carencia de estabilidad económica que nace el emprendedor,que en muchos casos, está dispuesto a arriesgar su idea en el mercado y sólo se ve impulsado por la necesidad, y en otros casos, con la ferviente creencia que el modelo innovador puede tomar vuelo frente al clima dinámico, cambiante y atractivo de la economía.

\section{¿Por qué emprender?}

"El Emprendimiento es un proceso que con el transcurso del tiempo ha venido causando un impacto positivo en la creación de empresas, fenómeno que coadyuva a la generación de empleos, mejorando su eficiencia en la productividad, hasta lograr un nivel de competitividad en mercados nacionales e internacionales." (Sparano, 2014, p. 96).

El emprendedor es la persona que posee criterios y capacidades que permiten que pueda arriesgarse a la aventura del emprendimiento. Así vemos que en el modelo de Collins las características que el sujeto presenta están ligadas a: la permanente motivación del logro, preferencia por el riego alto o moderado, preferencia por actividades que requieren esfuerzo, necesidad de actividades innovadoras, tendencia a planificar para el futuro, preferencia por los resultados individuales y atracción por un ambiente laboral acorde a la personalidad (Collins, Hanges, y Locke, 2004).

Mavila y otros autores también señalan que algunas características particulares de los emprendedores son: toma de riesgos, demanda por calidad y eficiencia, persistencia, búsqueda de oportunidades, establecimiento de metas, búsqueda activa de información, planificación sistemática y de control, capacidad creativa, entre otras (Mavila, Tinoco, y Campos, 2009).

1 Bachiller Andrea Teresa de Jesús Condori González. Administradora. Moda Shop E.I.R.L. E-mail: andrea_teresacg@hotmail.com

2 Magister Pedro Pablo Rosales López. Docente Investigador. USIL.

E-mail: pedro.rosales1@usil.pe 
Al respecto también se señala que desde niños no existe diferencia en la capacidad de motivación intrínseca y esta sólo varía por la edad. (Metallidou y Vlachou, 2007), lo que sugiere que todos tenemos la capacidad de emprender por convicción personal.

Se hace referencia al emprendedor como un tomador de riesgo, pero éste también puede ser identificado como la persona cuya característica principal es la de buscar mercados que están subvaluados para desarrollar su idea o invento. Entonces, el emprendedor es un "buscador" de oportunidades para nuevos negocios, que está alerta a las necesidades insatisfechas del mercado (Gómez, s.f.).

A nivel global, la intensidad de la innovación más bajo se da en África (20\%) y más alto en América del Norte (39\%). África tanto como América Latina y el Caribe muestran los niveles más altos de actividad emprendedora juvenil (16\%), América del Norte muestra tasas superiores al $10 \%$ en este grupo de edad. Por su parte América Latina y el Caribe tienen la mayor proporción de emprendedores entre los 45 - 54 y de 55- 64 años de edad (Global Entrepreneurship Monitor, 2017).

En América Latina se observa que el $42 \%$ de los emprendedores identifican oportunidades de negocios, mientras que el $28 \%$ se convierten en emprendedores por necesidad. Sin embargo, existen factores que se consideran esenciales tales como la gestión educativa que manejan los países, el apoyo del Estado a través de sus políticas para empresas sobre los impuestos, el apoyo financiero para la disponibilidad de recursos, préstamos y subsidios.

Las mujeres tienen más probabilidades de iniciar negocios por necesidad, en comparación con los hombres, y se ve así en tres regiones (América Latina y el Caribe, África y Europa).

Se sabe que hasta el año 2014 los países de América Latina que han causado últimamente un impacto positivo en el campo del emprendimiento han sido: Perú, Chile, Brasil, Argentina, Uruguay, Colombia, y México.
Para el año 2016, sesenta y cinco países que corresponden al $69.2 \%$ de la población actual participaron en el estudio de Global Entrepreneurship Monitor (2017) donde las variables analizadas fueron la actitud social con respecto al emprendimiento, las característica, motivación y ambiciones del emprendedor y la calidad de ecosistemas para el emprendedor en las diversas economías. Según dicho análisis, nuestro país Perú se encuentra catalogado como "Impulsado por la eficiencia", sin llegar aún a la clasificación que lo comprometa con un desarrollo óptimo en la innovación, caso contrario como el de Puerto Rico en la región. Los que corresponden a la categoría "Impulsado por la eficiencia" tienen las siguientes características con respecto a las percepciones sobre el emprendimiento:

- $42 \%$ Percibió oportunidades

- $55 \%$ Percibió aptitudes y capacidad propia

- $33 \%$ Tuvo miedo de fallar

- $26 \%$ Tiene intención de emprender un negocio dentro de 3 años como máximo

\section{Modelo de las fases del negocio según las ca- racterísticas del emprendimiento:}

En la tabla 1 se muestra la categorización de etapas del modelo emprendedor según Global Entrepreneurship Monitor, en donde diferentes variables afectarán el trance entre estas. En primera instancia la concepción, luego el nacimiento y finalmente la persistencia.

Según la perspectiva de Churchill y Lewis, las etapas del modelo de emprendimiento son: existencia, sobrevivencia, éxito, despegue y madurez de recursos.

Bajo esta perspectiva se puede concluir que el proceso es más largo de lo que muchos piensan, tras la etapa de sobrevivencia aún no está determinado si perdurará en el tiempo. Esto sólo será determinante al obtener una madurez en los recursos (Churchill y Lewis, 1983).

Tabla 1. Etapas del modelo de emprendimiento

\begin{tabular}{|c|c|c|c|c|}
\hline Etapa & Categorías & Características & \multicolumn{2}{|c|}{ Valores } \\
\hline 1 & Potencial & Oportunidades, conocimientos y habilidades & \multirow[b]{2}{*}{ Concepción } & \\
\hline 2 & Naciente & Involucrado en poner un negocio & & \multirow[b]{2}{*}{ Nacimiento } \\
\hline 3 & Propietario de un nuevo negocio & Menos de 3 años y medio & \multirow[b]{2}{*}{ Persistencia } & \\
\hline 4 & Propietario de un negocio estabilizado & Más de 3 años y medio & & \\
\hline
\end{tabular}

Fuente: Elaboración propia con datos de GEM 
Según la clasificación de Global Entrepreneurship Monitor (GEM) , en el Perú los aventureros en emprendimiento presentan los siguientes rangos de edad en promedio, siendo mayor afán por emprender de los grupos de edad entre los 25 a 34 años (17.5\%), seguido por los que oscilan entre 35 a 44 años (16.6\%).Luego le sigue el grupo de 45 a 54 (12.9\%) años A continuación siguen los más jóvenes entre 18 a 24 años (12.3\%) y finalmente, sólo un 9\% representa a los del rango entre 55 a 64 años.

\section{Impacto del emprendimiento}

Dependiendo de la etapa en donde se encuentra clasificada la economía del país (en el caso de Perú corresponde a la categoría "Impulsado por la eficiencia") la generación de oportunidades laborales es la siguiente:

- $46 \%$ no genera trabajo

- $35 \%$ genera de 1 a 5 trabajos

- $19 \%$ genera 6 a más trabajos

Resulta importante definir y cuantificar en valor monetario la representación de esta información para efectos en las mejoras de las políticas para el emprendimiento.

\section{El papel de los stakeholders para el desarrollo del emprendimiento}

Los actores del denominado Cuádruple de Hélix a nivel nacional (nivel académico, sector empresarial ,el Gobierno y la sociedad civil) desempeñan un papel importante para resaltar las formas en que la educación e instituciones pueden dotar a los individuos de un conjuntos de habilidades y hacer uso de oportunidades, así como la importancia de la Infraestructura informática eficiente para reducir el costo del negocio, incrementar la brecha del mercado, mejorar el acceso a la información y permitir la innovación.

A nivel académico la universidad es la entidad que propiciará adecuadamente las pautas para motivar el emprendimiento. El modelo que se propone según Castro es el de una universidad relacional, que tiene como principio la enseñanza articulada a competencias hacia el sentido de la iniciativa y el emprendimiento, un contexto aplicativo en lo económico, social, cultural y regional (Castro, Barrenechea, y Ibarra, 2011, p 208).

Las alianzas estratégicas universidad-estado se constituyen en una alternativa clave para la ejecución de proyectos de desarrollo empresarial (Duarte y Ruiz, 2009).
Las incubadoras han formado parte estratégica en el proceso de emprendimiento debido a que son articuladores activos entre los emprendedores y las fuentes de financiamiento, así como de la transferencia de tecnología. "De este modo las incubadoras cumplen el papel de empresas matriz, ya que abrigan las ideas de los emprendedores y se enfocan en darle forma, desde sus inicios, invirtiendo principalmente en los estudios necesarios para la transferencia y la comercialización de tecnologías" (Peña-Vinces, Bravo, Álvarez y Pineda, 2011, p. 15).

Es indispensable impulsar iniciativas que permitan cambiar la mentalidad de la sociedad estudiantil y migrar de una concepción tradicional del empleo hacia una transformación radical en la perspectiva laboral orientada a la creación de empresas, este rol resulta importante evidenciarlo en las entidades educativas que pueden apoyar al proceso de emprendimiento acelerado (Universidad Autónoma de Tamaulipas, 2006, p. 22).

\section{No rendirse jamás y volver a comenzar}

"Reset Economy es una categoría de análisis económico que analiza el comportamiento de las empresas y otras organizaciones que, tras atravesar un período de dificultades estructurales (es decir, no meramente coyunturales y debidas a factores como las fluctuaciones en la demanda, etc.), se replantean sus objetivos y su funcionamiento para conseguir un modelo de entidad económicamente sostenible" (Antuñano Maruri, I. y González Díaz, 2012, p. 53).

"Turnaround es el proceso de reconversión de una organización no rentable o insolvente en una organización rentable, solvente y por tanto sostenible; $y$ turnaround management es la parte de la gestión empresarial que se especializa en aplicar procesos de reconversión empresarial. En consecuencia podemos afirmar que el concepto de reset economy pone el acento en el resultado final consistente en la recuperación activa de una entidad, mientras que el concepto de turnaround se centra en el proceso de análisis y toma de decisiones que conduce a tal recuperación." (Antuñano Maruri, I. y González Díaz, 2012, p. 61).

La escalabilidad se podría definir como la habilidad de mantener un similar o mayor desempeño en el modelo de negocio, sea incrementando o reduciendo los componentes y recursos. Es esta característica importante para predecir la sostenibilidad que tendrá el negocio de emprendimiento a lo largo del tiempo. Muchos emprendimientos se dan por derrotados tras no haber considerado el factor que 
resume su trascendencia con un plan de contingencia para crecer en sus diferentes etapas sin perder usuarios, disminuir la calidad o cambiar algún valor en la empresa (Cavazos y Giuliani, 2016).

\section{METODOLOGÍA}

El enfoque de la investigación es cuantitativo, por la naturaleza de las variables que se utilizan.

A continuación, se muestra el procedimiento de elaboración de una encuesta dirigida a los estudiantes de la Universidad Nacional Mayor de San Marcos situada en Lima de diferentes escuelas de pregrado y postgrado con la intención de medir el impacto de su potencial emprendedor y su conocimiento sobre el tema e implicancias en general.

El instrumento utilizado, fue una encuesta respondida por estudiantes de la Universidad Nacional Mayor de San Marcos, de diferentes carreras y de diferentes facultades que pertenecen a pregrado y postgrado, que se encontraban en la Ciudad Universitaria, en la semana que se tomó la muestra. El tamaño de la muestra fue 100 .

El muestreo fue por conveniencia, ya que se aplicó la encuesta a los estudiantes que se encontraban en la Ciudad Universitaria, pero de todas las encuestas tomadas, en forma aleatoria se consideraron sólo cien encuestas. El instrumento fue validado por juicio de expertos, tanto en claridad como coherencia, en las dos dimensiones; logro motivacional y emprendimiento, y relación impacto en la economía. Tres docentes, del Instituto de Emprendedores de la USIL, fueron los jueces que colaboraron en este sentido. La confiabilidad del instrumento, fue corroborado en dos pilotos previos, el primer piloto fue tomado a 20 estudiantes de postgrado y el segundo piloto fue tomado a 50 estudiantes de pregrado y postgrado, el alpha de cronbach alcanzado fue de 0,75 .

La encuesta aplicada estuvo conformada por diez preguntas, agrupadas en dos dimensiones, como se muestra a continuación en la tabla 2 y la tabla 3 respectivamente:

Las dimensiones se tomaron en cuenta, son de un artículo de investigación que menciona explícitamente las características mínimas requeridas para el perfil de un emprendedor en "The Relationship of Achievement Motivation to Entrepreneurial Behavior : A Meta-Analysis".

Luego se ha procedido a aplicar la encuesta a los cien estudiantes seleccionados de manera aleatoria durante dos días en las instalaciones de la Universidad Nacional Mayor de San Marcos.

\section{RESULTADOS}

\section{Análisis de potencialidad emprendedora en los estudiantes de la Universidad Nacional Mayor de San Marcos}

En la figura 1 se muestra el rango de edades de las personas encuestadas para el análisis. La mayoría de ellos (46\%) corresponde al rango de 20 a 23 años de edad, seguido por un $26 \%$ que corresponde al rango de entre 17 a 20 años de edad. Se

Tabla 2. Dimensión logro motivacional y emprendimiento

\begin{tabular}{|l|}
\hline I. Relación logro motivacional y emprendimiento \\
\hline 1. Prefieres actividades que involucren habilidades y esfuerzo \\
\hline 2. Prefieres lidiar con riesgo moderado o alto riesgo \\
\hline 3. Prefieres involucrarte en actividades enérgicas e innovadoras \\
\hline 4. Prefieres planificar para el futuro \\
\hline 5. Prefieres el resultado de la tarea por responsabilidad individual \\
\hline 6. Prefieres que el ambiente de trabajo vaya de acuerdo a tu personalidad \\
\hline
\end{tabular}

Fuente: Elaboración propia

Tabla 3. Dimensión relación impacto en la economía

\begin{tabular}{|l|}
\hline II. Relación impacto en la economía \\
\hline 7. Consideras importante la formalización para tu emprendimiento \\
\hline 8. Conoces el procedimiento de formalización \\
\hline 9. Consideras importante los beneficios laborales de los trabajadores \\
\hline 10. Cuál será el rubro de tu emprendimiento \\
\hline 11. Cuántos trabajadores ( incluyéndote ) tendrá tu empresa \\
\hline
\end{tabular}

Fuente: Elaboración propia 


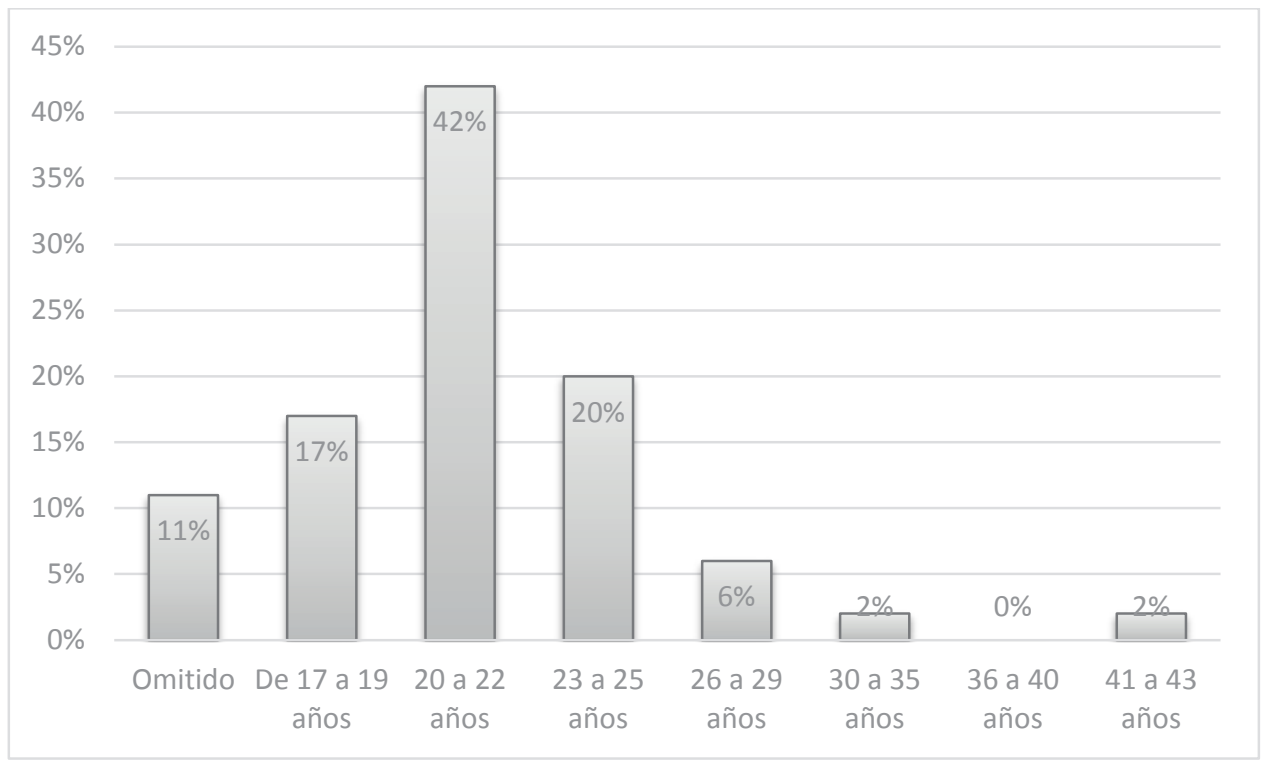

Figura 1. Rango de edades de alumnos encuestados de la UNMSM Fuente: Elaboración propia

0\% 10\% 20\% 30\% 40\% 50\% 60\% 70\% 80\% 90\%100\%

1.Prefieres actividades que involucren habilidades y esfuerzo

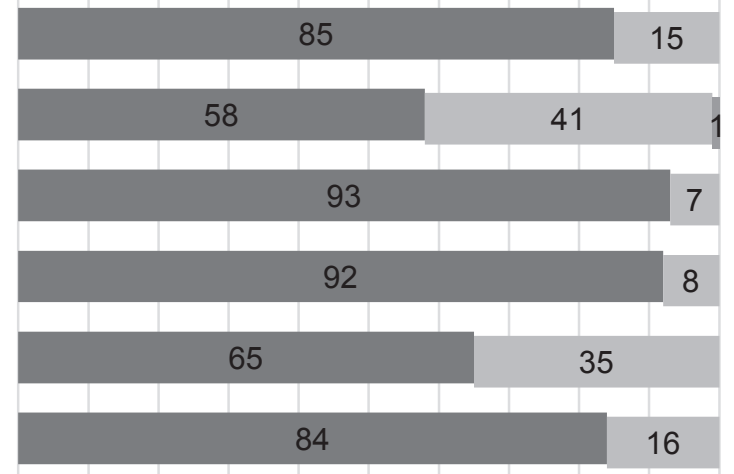

6.Prefieres que el ambiente de trabajo vaya de acuerdo a tu personalidad

con riesgo moderado o alto riesgo

3.Prefieres involucrarte en actividades enérgicas e innovadoras

4.Prefieres planificar para el futuro

5.Prefieres el resultado de la tarea por responsabilidad individual

\section{-RESPUESTAS SI $\square$ RESPUESTAS NO RESPUESTAS NS}

Figura 2. Resultados I parte relación logro motivacional y emprendimiento Fuente: Elaboración propia

evidenció un grupo equivalente al $11 \%$ que omitió información y un grupo muy pequeño que corresponde a un rango mayor de edad.

Los resultados de las dos dimensiones se muestran a continuación:

\section{Relación con logro motivacional y emprendi- miento:}

Se conoce que los factores relacionados con la edad ("age"), seguridad de la persona ("suskill") y el contexto emprendedor ("opport") son, a su vez, los factores de mayor influencia en la conducta de una persona frente al riesgo de emprender un nuevo negocio en América Latina. (Samaniego y Reyes, 2016). Estos factores se pueden traducir en la primera dimensión del experimento con la aplicación del cuestionario.

Al concluir el primer análisis de las seis primeras preguntas se tiene en la figura $\mathrm{N}^{\circ} 2$ un alto porcentaje de emprendedurismo en la muestra encuestada basándose en la potencialidad por el perfil de los encuestados, lo que corresponde al porcentaje promedio de $79.5 \%$ del potencial sobre la capacidad emprendedora en relación al logro motivacional y emprendimiento. 
Las variables que se reafirman con esta parte del cuestionario son la autonomía, seguridad personal, capacidad de respuesta personal, planificación a largo plazo, automotivación, rendimiento laboral, tolerancia al riesgo alto y orientación a la competitividad.

El $84 \%$ de encuestados respondieron que tienen predilección por un entorno más adecuado a su personalidad.

El $65 \%$ respondió que tiene preferencia por los resultados a partir del mérito propio.

El 92\% indicó que están más orientados a la planificación a largo plazo.

El 93\% de ellos prefiere involucrarse en actividades dinámicas: enérgicas e innovadoras.

El $58 \%$ de los encuestados tiene afinidad con la elección del riesgo en nivel moderado o alto.

Finalmente, el $85 \%$ de los alumnos encuestados optan por actividades que involucren sus habilidades y esfuerzo.

\section{Relación Impacto en la Economía}

Por su parte, en la figura 3 se presentan los resultados de la relación del impacto en la economía peruana. Los resultados en promedio son favorables dando como respuesta $73.67 \%$ de impacto positivo en la formalización tomando como referencia el conocimiento sobre los beneficios laborales, el procedimiento de formalización y la aplicación de la formalización en su modelo de negocio emprendedor.

Al analizar las variables de manera independiente se puede observar que el $97 \%$ de los encuestados considera muy importante los beneficios laborales de los trabajadores y estarían probablemente, bajo un esquema de trabajo justo para sus empleados. Sólo el $3 \%$ restante de ellos no lo considera trascendental.

Sobre el conocimiento del proceso de formalización, se evidencia la existencia de un porcentaje mucho menor que el promedio correspondiente a esta segunda dimensión, siendo $37 \%$ la totalidad de personas que respondieron afirmativamente y $62 \%$ las respuestas negativas sobre esta interrogante. Esto puede explicar el porqué de que nuestro país mantenga un alto índice de informalidad de la mano con gran cantidad de empresas bajo el régimen Mype. INEI también postula que a la fecha tenemos un aproximado de 6,6 millones de empresas o unidades productivas en el sector informal de la economía peruana (Saavedra, 2016) lo que reafirma esta respuesta.

A la pregunta sobre la necesidad que estarían dándole a la variable de formalización a sus emprendimientos, el $87 \%$ de los encuestados lo considera importante.

Los encuestados también mencionaron el área de emprendimiento en el que se van a desempeñar, siendo el área de "Tecnología" el que cuenta con mayor representación equivalente al $13 \%$, no necesariamente siendo su especialidad. Un $8 \%$ corresponde al área "Textil". El $6 \%$ corresponde a su preferencia por el campo "Legal". Un 5\% también ve representado su interés en un campo más amplio definido como "Ciencia y Tecnología", como se evidencia en la figura 4.

Esta afirmación responde a los cambios evolutivos de la globalización, la influencia de la era digital, las habilidades y destrezas de los millennials o generación naciente entre el siglo $\mathrm{XX}$ y principios del siglo XXI y la adaptabilidad propia de esta generación a los cambios tecnológicos.

0\% $10 \% 20 \%$ 30\% 40\% 50\% 60\% 70\% $80 \% 90 \% 100 \%$

7. Consideras importante la formalización para tu emprendimiento

8.Conoces el procedimiento de formalización para las empresas

9.Consideras importante los beneficios laborales de los trabajadores

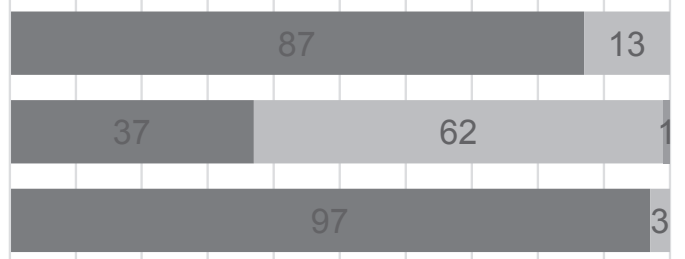

-RESPUESTAS SI -RESPUESTAS NO RESPUESTAS NS

Figura 3. Resultados II parte relación impacto en la economía Fuente: Elaboración propia 


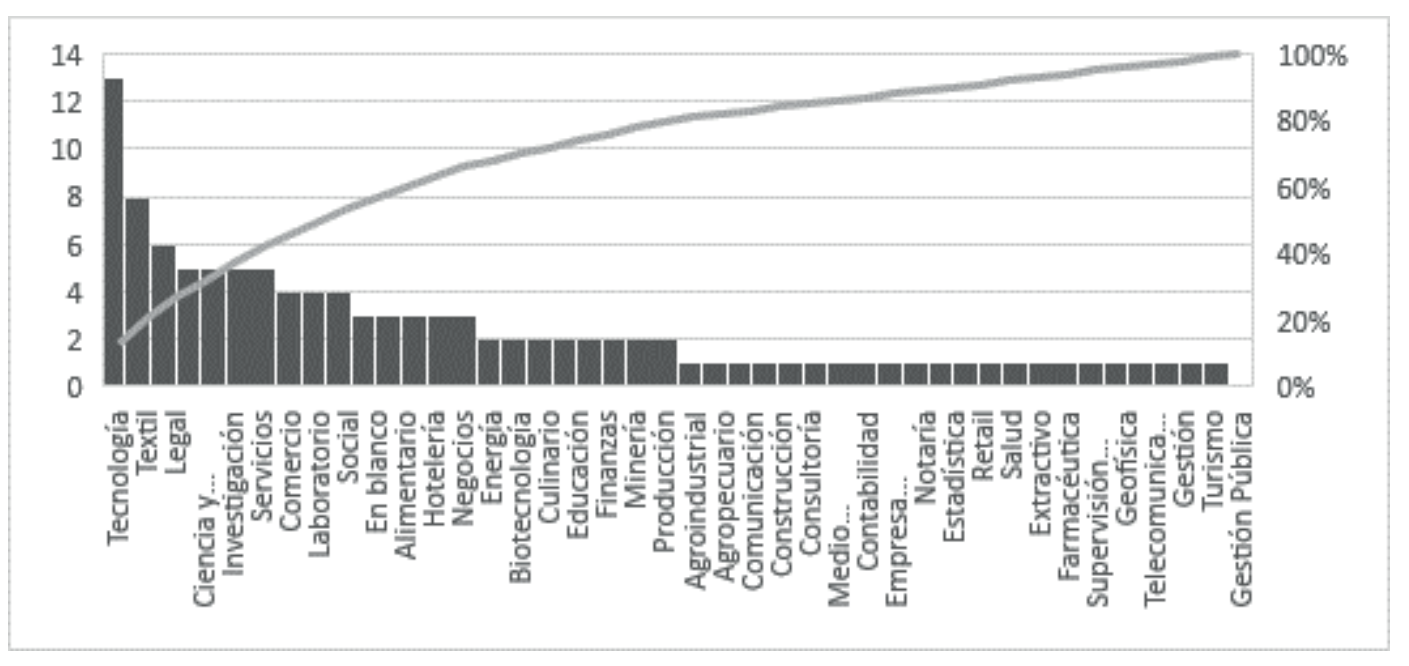

Figura 3. Resultados II parte relación impacto en la economía Fuente: Elaboración propia

Tabla 4. Áreas de interés en emprendimiento de los encuestados de la UNMSM

\begin{tabular}{|c|c|c|}
\hline Área de emprendimiento & Respuestas & $\mathrm{HI} \%$ \\
\hline Agroindustrial & 1 & $1 \%$ \\
\hline Agropecuario & 1 & $1 \%$ \\
\hline Alimentario & 3 & $3 \%$ \\
\hline Biotecnología & 2 & $2 \%$ \\
\hline Ciencia y Tecnología & 5 & $5 \%$ \\
\hline Comercio & 4 & $4 \%$ \\
\hline Comunicación & 1 & $1 \%$ \\
\hline Construcción & 1 & $1 \%$ \\
\hline Consultoría & 1 & $1 \%$ \\
\hline Contabilidad & 1 & $1 \%$ \\
\hline Culinario & 2 & $2 \%$ \\
\hline Educación & 2 & $2 \%$ \\
\hline Empresa Propia & 1 & $1 \%$ \\
\hline Energía & 2 & $2 \%$ \\
\hline Estadística & 1 & $1 \%$ \\
\hline Extractivo & 1 & $1 \%$ \\
\hline Farmacéutica & 1 & $1 \%$ \\
\hline Finanzas & 2 & $2 \%$ \\
\hline Geofísica & 1 & $1 \%$ \\
\hline Gestión & 1 & $1 \%$ \\
\hline Gestión Pública & 1 & $1 \%$ \\
\hline Hotelería & 3 & $3 \%$ \\
\hline Investigación & 5 & $5 \%$ \\
\hline Laboratorio & 4 & $4 \%$ \\
\hline Legal & 6 & $6 \%$ \\
\hline Medio Ambiente & 1 & $1 \%$ \\
\hline Minería & 2 & $2 \%$ \\
\hline Negocios & 3 & $3 \%$ \\
\hline Notaría & 1 & $1 \%$ \\
\hline Producción & 2 & $2 \%$ \\
\hline Retail & 1 & $1 \%$ \\
\hline Salud & 1 & $1 \%$ \\
\hline Servicios & 5 & $5 \%$ \\
\hline Social & 4 & $4 \%$ \\
\hline Supervisión/Control & 1 & $1 \%$ \\
\hline Tecnología & 13 & $13 \%$ \\
\hline Telecomunicaciones & 1 & $1 \%$ \\
\hline Textil & 8 & $8 \%$ \\
\hline Turismo & 1 & $1 \%$ \\
\hline En blanco & 3 & $3 \%$ \\
\hline Total & 100 & $100 \%$ \\
\hline
\end{tabular}

Fuente: Elaboración propia 
Sobre esta población Medina define que "actualmente en Latinoamérica $30 \%$ de la población es millennial. Además, según una proyección de la consultora Deloitte, en 2025 representarán $75 \%$ de la fuerza laboral del mundo". (Medina, 2016). Con esta información se puede deducir que, en efecto, la tecnología seguirá liderando las respuestas a medida que se incremente la muestra de encuestados.

Paradójicamente a lo que viene aconteciendo en el panorama social y el inminente cambio negativo del sector textil, se observa que en segundo lugar se encuentra la preferencia por los modelos de negocio en torno al sector textil. El 8\% afirmó que sus negocios de emprendimientos estarán dirigidos hacia esta dirección, como se evidencia en la figura 4.

El panorama en el sector textil no ha sido favorable en los últimos años siendo evidente que ha pasado por dificultades debido a la crisis financiera internacional, incluyendo también la cadena de valor y a los confeccionistas (Gestión, 2017).

En la figura 5 se muestran los rangos de números de plazas laborales potenciales, bajo la premisa de disponer actualmente de capacidad financiera y ejercer efectivamente el emprendimiento. Es asi que un $30 \%$ solicitará de 6 a 10 trabajadores, luego un $24 \%$ contratará de 16 a 25 trabajadores. Los "mono-negocios" constituidos por una sola persona o por dos son casi inexistentes en la muestra, siendo sólo un $2 \%$.
A modo de reflexión se puede mencionar que mantener de 6 a 10 trabajadores supone un presupuesto inicial considerable que muy probablemente será financiado por un tercero o por un capital inicial mayor, ajustando así la brecha de supervivencia.

\section{CONCLUSIONES Y RECOMENDACIONES}

- El emprendimiento es un fenómeno que ha sido inclusivo en diferentes ámbitos: de género y de finalidad, ya sea lucrativa o social. Mientras que responda adecuadamente a las necesidades reales del mercado tendrá mayor indicio de supervivencia. No se debe pasar por alto el modelo efectivo del emprendimiento, como lo es por oportunidad y no por necesidad si es que se busca mantenerlo a largo plazo. Tampoco se puede dar menor valor al rol de la mujer en este proceso ya que por sus características aporta más empatía a las necesidades insatisfechas del mercado y la convierte así en un actor cada vez con mayor participación.

- El Estado, más allá de implementar las políticas correctas para potenciar la formalización, sólo juega un papel pasivo frente a los resultados, que no serán los mejores, dada la carencia de influencia en todo el grupo de entidades colaborativas para el proceso de emprendimiento.

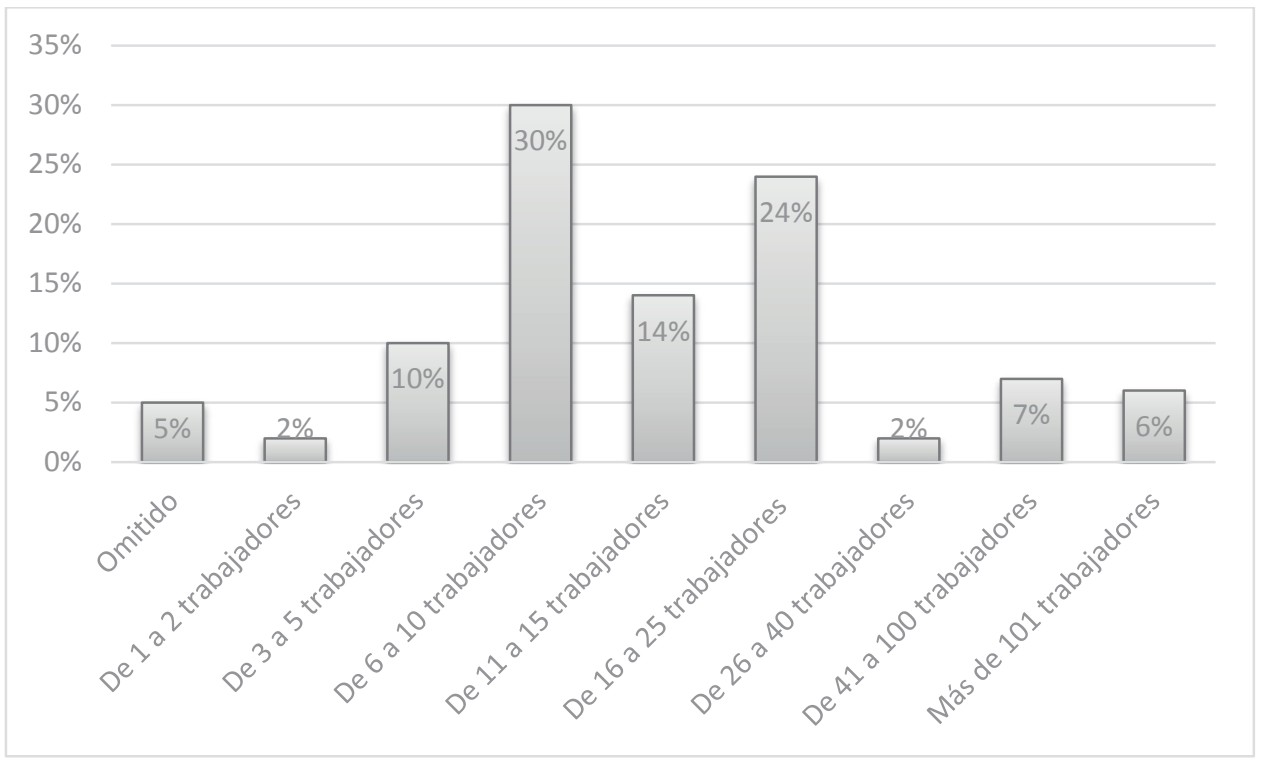

Figura 4. Capacidad de empleabilidad potencial en los emprendedores encuestados de la UNMSM Fuente: Elaboración propia 
- Las áreas de principal atención para los futuros emprendimientos en la Universidad Nacional Mayor de San Marcos son la de ciencia y tecnología, textil, legal, servicios e investigación. Para desarrollar óptimamente estos emprendimientos se necesitará considerable inversión inicial que debería ser facilitada con asesoría y simplicidad para promover a la empresa privada.

- El papel que juegan los actores del denominado Cuádruple de Hélix a nivel nacional (Nivel académico, sector empresarial, el Gobierno, la sociedad civil) es el más importante ya que puede dotar a los individuos de un conjunto de habilidades técnicas y así acceder a oportunidades adecuadas.

- Los estudiantes de la Universidad Mayor de San Marcos poseen un potencial alto en su perfil emprendedor, sin embargo; el impacto en la economía puede no ser favorable al observar carencia de conocimiento sobre temas básicos que beneficien a los stakeholders, como lo es el procedimiento de formalización de empresas. La carencia de conocimiento sobre la formalización debe erradicarse bajo la supervisión del Estado o la Universidad, considerando el acercamiento hacia estos grupos juveniles para orientarlos de manera adecuada y así evitar la crisis de la informalización a largo plazo.

- Sobre las áreas de emprendimiento, surge la particularidad de observar en mayor número el área de Tecnología anidada con Ciencia y Tecnología, pudiendo explicarlo como la respuesta a una sociedad digitalizada e implementada de cambios tecnológicos continuamente.

- El papel de las incubadoras es fundamental, de la mano con el Estado, para definir cuál será el futuro real de la economía de nuestro país a futuro.

\section{REFERENCIAS BIBLIOGRÁFICAS}

[1] Antuñano Maruri, I. y González Díaz, A. (2012). Reset economy y economía social. CIRIECEspaña. Revista de Economía Pública, Social y Cooperativa, núm. 76, p. 51-73.

[2] Bravo, C. (2016). Las escuelas de pensamiento del emprendimiento social. TEC Empresarial.
[3] Castro Spila, J., Barrenechea, J., e Ibarra, A. (2011). Cultura emprendedora, innovación y competencias en la educación superior: el caso del programa Gaze. ARBOR Ciencia, Pensamiento y Cultura, (187), p. 207-212. doi:10.3989

[4] Cavazos, J., y Giuliani, A. (2016). Escalabilidad: Concepto, características y retos desde el emprendimiento comercial y social. Puebla.

[5] Churchill, N., y Lewis, V. (1983). The five stages of small business growth. Harvard Business Review.

[6] Collins, C., Hanges, P., y Locke, E. (2004). The Relationship of achievement motivation to entrepreneurial behavior: a meta analysis. Ithaca.

[7] Duarte, T., y Ruiz, M. (2009). Emprendimiento, una opción para el desarrollo. Emprendiendo en la era del conocimiento. (2006). Ciencia UAT.

[8] Gestión. (2017). El Gobierno seguirá apoyando a la industria textil. Gestión.

[9] Global Entrepreneurship Monitor. (2017). Global Report 2016/17.

[10] Gómez, A. (s.f.). Creadores de riqueza, emprendedores que cambiaron nuestras vidas.

[11] Mavila, D., Tinoco, O., y Campos, C. (2009). Factores influyentes en la capacidad emprendedora de los alumnos de la Universidad Nacional mayor de San marcos. Industrial Data. 12(2), p. 32-39.

[12] Medina, C. (2016). Los millennials su forma de vida y el streaming. Gestión y Estrategia.

[13] Metallidou, P., \& Vlachou, A. (2007). Motivational beliefs, cognitive engagement, and achievement in language and mathematics in elementary school children. International Journal of Psychology, 42(1), p. 2-15. Recuperado de https://onlinelibrary.wiley.com/ doi/abs/10.1080/00207590500411179

[14] Peña-Vinces, J., Bravo, S., Álvarez, F., y Pineda, D. (2011). Analysis of Characteristics of Business Incubators in Colombia: A Case Study. Journal of Economics, Finance \& Administrative Science, 16(30), p. 13-28. Recuperado de http://www.scielo.org.pe/pdf/ jefas/v16n30/a03v16n30.pdf.

[15] Saavedra, M. (Febrero de 2016). INEI: Los impresionantes números del sector informal peruano. El Comercio. 
[16] Samaniego Alcántar, A., \& Reyes Ruiz, G. (2016). Factors that influence the behavior of a person against the risk of starting a business in Latin America. Journal of Business Universidad del Pacífico, 8(1), p. 57-71. Recuperado de https://rei.iteso.mx/ bitstream/handle/11117/4159/Empezar\%20 un $\% 20$ negocio-factores $\% 20$ de $\% 20$ riesgoSamaniego $\% 20 y \% 20$ Ruiz.pdf?sequence $=4$

[17] Sparano, H. (2014). Emprendimiento en América Latina y su impacto en la gestión de proyectos. Revista Dimensión Empresarial, 12(2), p. 95-106.
[18] Universidad Autónoma de Tamaulipas. (2006). Emprendimiento en la era del conocimiento. Ciencia UAT, 1(2), p. 19-22. Recuperado de http://www.redalyc.org/articulo. oa?id=441942906009

[19] Vásquez, M., Camacho, G., y García, C. (2016). Women empowerment through social innovation in indigenous social enterprises. Mackenzie Management Review. 